\title{
Reflets
}

Revue d'intervention sociale et communautaire

\section{Pauvreté, inégalités de revenu et santé : quels liens? Entrevue avec Dennis Raphael}

\section{Dahlia Namian}

Volume 25, numéro 1, printemps 2019

Actualité de la pauvreté : débats théoriques, défis pratiques

URI : https://id.erudit.org/iderudit/1064665ar

DOI : https://doi.org/10.7202/1064665ar

Aller au sommaire du numéro

Éditeur(s)

Reflets, Revue d'intervention sociale et communautaire

ISSN

1203-4576 (imprimé)

1712-8498 (numérique)

Découvrir la revue

Citer ce document

Namian, D. (2019). Pauvreté, inégalités de revenu et santé : quels liens?

Entrevue avec Dennis Raphael. Reflets, 25(1), 20-28.

https://doi.org/10.7202/1064665ar d'utilisation que vous pouvez consulter en ligne.

https://apropos.erudit.org/fr/usagers/politique-dutilisation/ 


\section{Pauvreté, inégalités de revenu et santé : quels liens?}

\section{Entrevue avec Dennis Raphael}

Professeur, Université York

Cette entrevue a été réalisée le 7 décembre 2018 et traduite de l'anglais par

\section{Dablia Namian}

Professeure agrégée, École de service social, Université d'Ottawa

\section{Présentation}

Dennis Raphael, PhD est professeur de politique et de gestion de la santé à l'Université York à Toronto. Il a publié plus de 200 articles sur les déterminants sociaux de la santé en plus d'avoir publié et édité une vingtaine d'ouvrages sur la question, dont Staying alive: Critical perspectives on health, illness and health care (2010), Poverty in Canada. Implications for Health and Quality of Life (2011) et Determinants of Health: A Canadian Perspective (2016).

Reflets: Votre ouvrage collectif Determinants of health: A Canadian perspective (2016), a été réédité trois fois depuis sa sortie en 2004, et fait partie de la plupart des curriculums d'étude dans les écoles de travail social canadiennes. Pourquoi est-ce important de considérer la relation entre santé et pauvreté?

D. Raphael : Il existe une quantité considérable d'études sur la santé, mais la plupart d'entre elles abordent les " comportements » liés aux choix et modes de vie, par exemple, en abordant les liens entre l'alcool et la santé ou l'obésité et les choix de consommation. Ce qui est important à mon point de vue, c'est que les personnes pauvres et à faible revenu sont non seulement plus exposées aux risques de maladies et de mortalité prématurée, mais elles font 
aussi l'expérience de ces risques dans un environnement où le discours dominant les blâme de tomber malade et de mourir plus tôt, sans tenir compte des déterminants sociaux, tels que les conditions de travail, qui ont une influence sur leur santé (Raphael et collab., 2019a; Medvedyuk, Ali et Raphael, 2018). Donc, nous ajoutons l'injure à la blessure; non seulement nous plaçons les personnes pauvres dans une situation qui détériore leur santé, mais nous les insultons ensuite en leur disant qu'ils sont responsables de ce mauvais état de santé. Si je travaillais dans un département de sciences économiques ou politiques, je dirais la même chose, mais sans insister sur la santé. Certains disent que nous ferions mieux de ne pas parler de santé, mais d'inégalités et de violation des droits de la personne, car dès que vous parlez de santé, la tendance est de répondre au problème par la santé, par la modification des choix de vie, plutôt que par des initiatives agissant sur ses déterminants sociaux.

Reflets : Le Canada est souvent reconnu comme l'un des pays développés les plus riches dans le monde. En même temps, les chiffres tendent à montrer que les inégalités de revenus augmentent. Vous appuyant sur une forte analogie dans le titre d'un article [voir Raphael et Bryant, 2014], vous déclarez que les effets des inégalités de revenus au Canada sont à l'image de " 110 victimes d'écrasement d'avion, chaque jour, 365 jours par année ". Pouvez-vous expliquer cette analogie et comment les inégalités de revenus affectent la santé des Canadiennes et des Canadiens?

D. Raphael : La genèse de cette idée est de mettre un coût en dollars sur les inégalités. Dans un rapport publié par Statistique Canada, Tjepkema, Wilkins et Long (2013) affirment qu'en comparant la santé des $20 \%$ de Canadiennes ou Canadiens les plus riches à celle des $80 \%$ autres Canadiennes ou Canadiens, on trouve $40 \%$ de décès prématurés chez ces $80 \%$. De plus, si on examine le taux de mortalité prématuré toutes années confondues parmi les $20 \%$ les plus pauvres, comparativement aux $20 \%$ les plus riches au Canada, on constate que les $20 \%$ les plus pauvres ont environ $65 \%$ plus de risque de mourir de maladies, telles 
que le cancer et le diabète. Si l'ensemble des Canadiennes et Canadiens étaient en aussi bonne santé que les $20 \%$ les plus riches, il y aurait 40000 décès de moins par an. Nous sommes dans un contexte néolibéral où les inégalités sociales de la santé s'aggravent. Les $60 \%$ de Canadiennes ou Canadiens les moins bien nantis sont exposés à des risques croissants de santé liés notamment à la stagnation ou la réduction des salaires, à la croissance des inégalités de revenu, à la précarité croissante du travail et à la crise du logement. Dans l'ouvrage Determinants of Health: A Canadian Perspective cité plus haut, on observe que les inégalités de santé concernent particulièrement les groupes socialement marginalisés. Ces inégalités sont par exemple beaucoup plus significatives chez les populations autochtones comparativement aux populations non autochtones.

Reflets : Vous avez commencé à travailler sur les inégalités de santé dans les années 1990, alors que nous observions une forte dérèglementation de la main-d'euvre, parallèlement à une réduction importante des politiques en matière de protection sociale. Depuis lors, l'insécurité de l'emploi augmente au Canada et nous sommes maintenant confrontés à un nombre croissant de travailleurs pauvres dans une économie en mutation rapide. Bien que le projet de revenu universel ne soit plus à l'ordre du jour en Ontario, devrait-il être considéré comme une solution viable à la pauvreté?

D. Raphael : Dans les années 1990, on commence à observer des changements dans les politiques publiques et vers le milieu des années 1990, on observe une plus forte tendance à la mondialisation et à la circulation des capitaux, qui donne plus de pouvoirs au secteur des entreprises. Le Canada, qui est en principe une économie politique libérale, a participé à approfondir les tendances à la déréglementation du marché et à la privatisation des secteurs sociaux et de santé. Au cours des années 1990, on commence donc à observer un ensemble de compressions des dépenses dans les programmes sociaux, telle l'annulation du programme fédéral de logement; au même moment, on a accordé des centaines d'allégements fiscaux aux entreprises. Il n'est donc pas 
surprenant que le gouvernement canadien affirme qu'il n'a pas assez d'argent pour la santé et d'autres services publics. Vers le début des années 2000, bien qu'on commence à se soucier des déterminants sociaux de la santé, on ne fait pas le lien entre les inégalités en matière de santé et l'accroissement du pouvoir des entreprises. De plus, si beaucoup de publications portant sur les déterminants sociaux de la santé déclarent que pour améliorer leur santé les Canadiennes et Canadiens ont besoin d'un système universel de garderie pour les enfants, de formations à l'emploi et d'emplois moins précaires ou de logements plus accessibles, elles ne sont pourtant pas en mesure de lier ces mêmes déterminants sociaux à la littérature sur l'économie politique qui souligne le pouvoir croissant des entreprises (Raphael et collab., 2019b). Or cette littérature, (voir, entre autres Brennan (2012), indique clairement les liens directs entre le déclin des salaires et la concentration du pouvoir des entreprises. À cet effet, j'ai coécrit un article (Mendley-Zambo et Raphael, 2018) sur l'insécurité alimentaire dans lequel nous expliquons que si les inégalités en matière de santé sont liées au faible niveau d'assistance sociale, aux bas salaires ou au manque de logements abordables, il y a une incapacité à reconnaître comment ces inégalités sont intimement liées aux écarts de revenu et aux $20 \%$ les plus riches.

Cela nous mène à nous poser la question : quelle est la solution? Beaucoup de gens comme moi disent qu'il faut des syndicats plus forts et un rééquilibrage du pouvoir entre l'État, la société civile et le secteur des entreprises. D'autres personnes vont insister sur un revenu universel grâce auquel on serait capable d'éliminer les sources les plus flagrantes de pauvreté. En Ontario, si on vous considère " apte à l'emploi ", mais que vous recevez de l'aide sociale, vous recevez environ 7000 \$ par an, ce qui, bien évidemment, est une recette pour la maladie et la mort. Le revenu universel vous permettrait de passer alors de 7000 \$à $14000 \$$ ou 15000 \$ par an (Raphael et collab., 2018). Cette solution peut certainement empêcher les gens de tomber à la rue, voire de mourir de faim. Or la mise en place d'un revenu universel ne va 
pas pour autant à la source du problème, à savoir, les bas salaires et le déséquilibre des pouvoirs dans la société. Donc, même si on met en place un revenu de base, il y a selon moi deux problèmes. Premièrement, les personnes resteront sous le seuil de pauvreté, de sorte qu'elles seront entre autres toujours à plus haut risque de maladie. Deuxièmement, cette mesure peut en venir à détourner l'attention des véritables causes de la pauvreté, à savoir les bas salaires, le travail précaire, le manque de logements et de garderies accessibles, etc. Ce qui est important à considérer, c'est que le revenu universel ne devrait pas se substituer à toutes ces choses. On s'intéresse de plus en plus au revenu universel au sein de la littérature universitaire, mais cette dernière ne remet pourtant pas en cause ces autres problèmes structurels, dont le travail précaire ou les bas salaires. La seule critique réelle ne vient pas du monde universitaire, mais de certains mouvements sociaux, notamment de l'Ontario Coalition against Poverty - OCAP (2017). Il s'agit selon moi d'une politique publique générale qui vient réduire le rôle de l'État dans la régulation de l'économie et ses dépenses sociales en termes de logements abordables, de formation professionnelle, de garderies, et cela, dans un contexte où le secteur des entreprises continue de supprimer les salaires et d'aggraver en toute impunité les conditions de travail. Si la tendance se maintient, nous observerons dans un proche avenir une régression ou une diminution constante des dépenses en programmes et en services publics.

Reflets: Vos recherches montrent que la qualité des conditions de vie qui façonnent la santé est fortement déterminée par les décisions prises par les gouvernements dans divers domaines de politique publique. Selon vous, quels programmes ou quelles politiques publiques semblent prometteurs pour réduire les inégalités de santé?

D. Raphael : Je pense que nous devrions nous intéresser davantage aux pays scandinaves. Ils offrent non seulement des programmes universels de santé, mais également des salaires plus élevés et une forme protégée de travail. Cela signifie que si vous travaillez, vous n'êtes pas pauvre et que $70 \%$ des travailleurs sont syndiqués. 
Cela tient également à la nature de la société, aux traditions historiques et à une conception solide de ce que devrait être une société. Au Québec, il y a un fort mouvement de femmes qui se sont battues notamment pour les garderies universelles, mais ce type de mouvement fait défaut dans le reste du Canada. Au Canada, cependant, la majorité des Canadiennes et Canadiens croient encore au rôle de l'État et de la communauté. Or si le parti Libéral tend à remporter ses campagnes à gauche, il a tendance à gouverner à droite. Le message que les Canadiennes et Canadiens reçoivent de l'État est que son rôle est de faire en sorte que les entreprises continuent de prospérer. Lorsque l'usine de General Motors a été fermée, le message qui a été transmis aux Canadiennes et Canadiens est que rien ne pouvait être fait. Eh bien! si vous regardez les pays scandinaves, vous voyez que certaines choses peuvent être faites et vous comprenez que l'État a la responsabilité de fournir une sécurité économique et sociale à la population. Dans les pays d'Europe du Nord, l'idéologie sousjacente est l'égalité et les politiques publiques sont soucieuses de réduire les inégalités. En Allemagne, en France, voire au Québec, l'idéologie sous-jacente est "solidaire " et cherche à garantir une certaine sécurité sociale. Dans les pays libéraux anglo-saxons, l'idéologie première est la liberté, et cette liberté se traduit par une liberté pour les entreprises qui ont le pouvoir de faire ce qu'elles veulent...

Reflets: Le travail social est une profession qui cuvre en étroite collaboration avec les communautés et les individus afin d'améliorer leurs conditions de vie. Les déterminants sociaux de la santé font partie intégrante de la pratique, même sìls ont encore du mal à se faire reconnaître dans un contexte où les ressources publiques sont de plus en plus rares en soins de santé. Selon vous, comment les travailleuses sociales et les travailleurs sociaux peuvent-ils être les plus utiles pour faire face aux impacts des inégalités de santé sur les communautés et sur les individus?

D. Raphael : Je me référerais aux articles de Bryson et Bosma (2018) qui soutiennent qu'aujourd'hui les travailleuses sociales et les 
travailleurs sociaux s'intéressent davantage aux déterminants sociaux de la santé. Le travail social est devenu plus actif dans le domaine de la santé, alors qu'environ 50\% des travailleuses sociales et travailleurs sociaux sont impliqués ou travaillent dans le secteur de la santé, traitant quotidiennement avec ces déterminants : pauvreté et revenu, logement, insécurité alimentaire, racisme, sexisme, etc. Maintenant, je pense que si le travail social reconnaît ces problèmes, la question demeure : peut-il faire quelque chose à leur sujet? Comme le soutient aussi Moniz (2010), les trois principales recommandations en termes d'action sur les déterminants sociaux de la santé sont d'améliorer des conditions de vie quotidienne, de s'attaquer à la répartition inégale des revenus, du pouvoir et des ressources, et de concentrer le travail sur le plan de la communauté et des politiques publiques.

Les travailleuses sociales et travailleurs sociaux constituent une partie importante de la société. Elles et ils possèdent non seulement l'expertise, mais également la capacité de résoudre publiquement les problèmes liés aux déterminants sociaux de la santé. À mon avis, le problème est que les déterminants sociaux de la santé portent le mot "santé » et que, lorsque vous parlez de santé, les professions médicales ont tendance à écarter le travail social... Comme toute autre chose, la santé est un domaine encombré; les médecins, les épidémiologistes, les infirmières et autres professions reliées à la santé ne veulent pas forcément que les travailleuses sociales et travailleurs sociaux aillent sur leur territoire. Or si vous regardez ce que les travailleuses sociales et travailleurs sociaux sont censés faire, leur rôle touche directement aux déterminants sociaux de la santé, même s'ils ne les appellent pas toujours ainsi. Encore une fois, le travail social a le potentiel d'influencer les pratiques quotidiennes, d'influencer le pouvoir des communautés et d'ouvrir une tribune aux associations et à toutes celles et à tous ceux qui militent pour la réduction de la pauvreté, l'accessibilité au logement ou l'amélioration des conditions de travail et du revenu. 


\section{Bibliographie}

BRENNAN, Jordan. (2012). A shrinking universe: How concentrated corporate power is shaping income inequality in Canada, Canadian Centre for Policy Alternatives, 50 p.

BRYSON, Stephanie A., et Harvey BOSMA (2018). " Health social work in Canada: Five trends worth noting ", Social work in health care, Vol. 57, No 8, p. 1-26.

MEDVEDDYUK, Stella, Ahmednur ALI et Dennis RAPHAEL (2018). "Ideology, obesity and the social determinants of health: a critical analysis of the obesity and health relationship ", Critical Public Health, Vol. 28, № 5, p. 573-585.

MENDLY-ZAMBO, Zsofia, et Dennis RAPHAEL (2018). "Competing discourses of household food insecurity in Canada ", Social Policy and Society, réf. du 10 mai 2019,

https://doi.org/10.1017/S1474746418000428

MONIZ, Cynthia (2010). "Social work and the social determinants of health perspective: A good fit ", Health and Social Work, Vol. 35, № 4, p. 310 - 314.

ONTARIO COALITION AGAINST POVERTY (OCAP) (2017). Basic Income in a Neoliberal Age, réf. du 15 avril 2019, https://socialistproject.ca/pamphlets/basic-income-neoliberal-age/

RAPHAEL, Dennis, Toba BRYANT et Marcia H. RIOUX (dirs.) (2010). Staying alive: Critical perspectives on health, illness, and health care, Toronto, Canadian Scholars' Press, 408 p.

RAPHAEL, Dennis (2011). Poverty in Canada: Implications for health and quality of life, Canadian Scholars' Press Inc., 522 p.

RAPHAEL, Dennis, et collab. (2019a) "The cultural hegemony of chronic disease association discourse in Canada ", Social Theory \& Health, réf. du 10 mai 2019, https://doi.org/10.1057/ s41285-018-0072-7

RAPHAEL, Dennis, et collab. (2019a). "The cultural hegemony of chronic disease association discourse in Canada ", Social Theory \& Health, p. 1-22.

RAPHAEL, Dennis, et collab. (2019b). "Governmental illegitimacy and incompetency in Canada and other liberal nations: Implications for health ", International Journal of Health Services, Vol. 49, No 1, p. 17-36.

RAPHAEL, Dennis, Toba BRYANT et Zsofia MENDLY-ZAMBO (2018). "Canada considers a basic income guarantee: can it achieve health for all? ", Health promotion international, Vol. 34, No2, p. 179-181.

RAPHAEL, Dennis, et Toba BRYANT (2014), réf. du 10 mai 2019, http://www.thinkupstream. net/health_effects_of_income_inequality\#_edn1

RAPHAEL, Dennis, et Toba BRYANT (2014). "The effects of income inequality: A jet with 110 Canadians falling out of the sky each day, every year, 365 days a year ", réf du 15 avril 2019, http://www.thinkupstream.net/health_effects_of_income_inequality\#_edn1 
RAPHAEL, Dennis (2016). Social determinants of health: Canadian perspectives, 3rd edition, Canadian Scholars' Press Inc., $624 \mathrm{p}$.

TJEPKEMA, Michael, Russell WILKINS et Andrea LONG (2013). " Cause-specific mortality by income adequacy in Canada: A 16-year follow-up study ", Statistic Canada, Health Reports, Vol. $24, \mathrm{~N}^{\circ} 7$, p. 14-22. 